\title{
Mejorando la experiencia del turismo cultural con un prototipo de realidad virtual
}

\author{
Gabriel Peralta Domínguez, Pedro C. Santana-Mancilla \\ HCLab \\ Facultad de Telemática \\ Universidad de Colima \\ \{peralta_gabriel, psantana\}@ucol.mx
}

\begin{abstract}
Resumen. Este artículo presenta una propuesta de prototipo que permite a los visitantes de un lugar realizar recorridos de corte turístico-cultural, por medio de un prototipo de mediana fidelidad con tecnología de Realidad Virtual. Se realizó una evaluación de usabilidad, donde el objetivo fue determinar el nivel en qué los usuarios pueden aprender y utilizar el sistema propuesto para alcanzar sus metas o realizar diversas tareas de esparcimiento en relación a ciertos recorridos propuestos. Además de evaluar la aceptación de dicho prototipo por parte de los usuarios.
\end{abstract}

Palabras clave: Realidad virtual, Turismo virtual, Experiencia del usuario.

\section{Introducción}

En mayor o menor medida la sociedad es testigo de las transformaciones impulsadas por las tecnologías, los desarrollos tecnológicos se han convertido en elementos imprescindibles, formando parte de una cotidianeidad que evolucionó históricamente sin comparación a partir de los últimos años del siglo XX. De estos adelantos, las Tecnologías de la Información y la Comunicación (TIC) son los ejemplos más evidentes. Una de las definiciones globales, formulada por la comisión de la Comunidad Europea en el año 2001 señala: Las tecnologías de la información y de la comunicación es un término que se utiliza actualmente para hacer referencia a una gama amplia de servicios, aplicaciones y tecnologías que utilizan diversos tipos de equipos (Hardware) y de programas informáticos (Software) y que a menudo se trasmiten a través de las redes de telecomunicaciones (Netware) [1].

Con las TIC disponemos de nuevos lenguajes y medios que permiten una trasformación en la forma de guardar nuestra memoria en su conservación y preservación, en las funciones de los centros culturales, en el proceso de comunicación, en la transmisión de conocimientos, en el acercamiento real al patrimonio..., si somos capaces de aprovechar sus potencialidades para re-crear nuevas formas, más allá de la falsa modernidad de un envoltorio vacío de contenidos, Unas potencialidades, aún infravaloradas, que nos plantean nuevos retos para el siglo XXI: creación de contenidos de calidad y generación de información innovadora para convertir la información en conocimiento, difusión del patrimonio de forma atractiva y amena orientada a la educación y al público en general, a la exploración científica 
de los profesionales y a la creación artística. El patrimonio rompe su exclusiva dimensión física lo que implica el acercamiento del patrimonio a la sociedad con una perspectiva diferente [2].

Por estas razones el patrimonio cultural no debe quedar supeditado a su apreciación y conceptualización tradicionales, por el contrario, dada nuestra realidad tecnológica, las TIC supone un excepcional potencial para la realización de tareas como la gestión, documentación, conservación, investigación, difusión y conocimiento. [3]

Es así, que actualmente pueden hallarse múltiples aplicaciones relacionadas como productos multimedia en diversos soportes, visitas virtuales a museos, itinerarios culturales, reconstrucciones de emplazamientos o edificios, contextualización de las expresiones culturales (espacial, temporal, social o cultural), etc.

Esta investigación se basa en dichos planteamientos, donde estas nuevas tecnologías facultan a los usuarios el acercamiento y reconocimiento de patrimonio, que dada su ubicación, distancia, costo de acceso, entre otras características que requiere la presencia personal para la apreciación de recintos de carácter artísticocultural, tienen una valor agregado también como capital turístico.

Dicho capital tiene un objetivo clave en el desarrollo económico de los sectores sociales involucrados, uno de los principales para dicho progreso, es el empleo, ya que el sector de las Nuevas Tecnologías de la Información y la Comunicación suponen nuevas oportunidades para el conjunto de los ciudadanos, pero también nuevas amenazas para los que no tienen una relación cercana con ellas. La creación de empleo ha beneficiado a los trabajadores de niveles educativos medios y altos y presenta también un sesgo positivo hacia dos de los colectivos con una situación más desfavorable en el mercado de trabajo. [3]

Este proyecto de aplicación, pretende favorecer a dichos sectores económicos vulnerables, como lo es la de los trabajadores de servicio de lustrado de calzado, conocidos en el argot coloquial como "boleros", siendo estos parte importante del paisaje urbano, íconos locales de un oficio tradicional y esencia de un pasado histórico de las ciudades que aun los resguarda.

Así que nuestra propuesta consiste en un prototipo de mediana fidelidad de recorrido virtual que utiliza las tecnologías de Google: Maps y Street View; y que sea capaz de realizar recorridos en $3 \mathrm{D}$ por los recintos culturales e históricos de la localidad, con un alto nivel de inmersión por medio del dispositivo de realidad virtual, con el fin de estimular la presencia de turistas en las estaciones de servicio de lustrado de calzado y con ello prestar un valor agregado a la prestación del servicio, ofreciendo al usuario, sin moverse de su asiento, la oportunidad de visitar de nueva forma alternativa, diferentes destinos considerados como patrimonio regional y de interés turístico.

\section{Contexto}

El diseño del prototipo está encaminado a un entendimiento más preciso sobre las actividades y actitudes realizadas por parte de los participantes observados, que corresponden a los trabajadores de lustrado de calzado, que de ahora en adelante 
llamaremos "boleros" y las características de los usuarios o "clientes" potenciales. El trabajo de campo se establece en el municipio de Ciudad Guzmán, Jalisco, una pequeña ciudad seleccionada por su reconocida riqueza cultural y cuna de célebres artistas y literatos. En ella, reside una de sus más importantes plazas públicas, el jardín central, donde en dicho paisaje urbano, los tradicionales "boleros" comparten espacio con otros recintos históricos colindantes. Dichos trabajadores independientes, realizan una labor típica de este lugar, la cual es lustrar el calzado de los visitantes del jardín principal. Donde desde hace décadas han mantenido su presencia en los alrededores del mismo sitio, por lo que su alcaldía busco mejorar las condiciones con las que se establecieron $\mathrm{y}$ formalizar su estancia. Por tanto, se asignaron y reacondicionaron espacios más atractivos y eficientes en diseño, además de exclusivos para establecerse de manera más permanente, donde según sus propios habitantes, fueron designados con el objetivo de mantener el oficio del "bolero", y que por su histórica presencia, se convirtieron en íconos y parte del patrimonio cultural intangible de esa localidad.

\subsection{Método}

Para fines de recolección de información sobre requerimientos y comportamiento de los usuarios, se empleó la observación directa (activa) hacia los boletos y sus clientes en el Jardín Principal, de Ciudad Guzmán, Jalisco; con un muestreo aleatorio para captar con mayor detalle la naturaleza de las situaciones, con el uso de un herramientas de grabación de notas de voz y cámara fotográfica. No se tipificó perfiles o características predeterminadas, ya que el propósito fue precisamente, el reconocimiento de sus probables perfiles, sin embargo, se consideraron aspectos como la ubicación del puesto de servicio, su tamaño (si este se extendía), la variabilidad de carga de trabajo según horario, sus servicios o productos secundarios ofertados, así como los elementos estéticos que pueden intervenir en su elección, como la organización, higiene y estética, publicidad, y otros posible de detectar durante la observación.

\subsection{Resultados}

Se observaron las características del puesto donde se encuentran ubicados los boleros. En total son 16 puestos ubicados en la parte oriente y poniente del Jardín. Cada puesto consta de una caseta con capacidad para dos sillas de bolero, las sillas están sobre una tarima de madera, en la cual se encuentran cajones para el almacenamiento de los utensilios y materiales que se utilizan para bolear calzado.

En la parte del frente de cada caseta, están instaladas lonas para tapar la luz del sol, que puede ser molesta debido a la ubicación de los puestos. Dichas lonas tienen impresas imágenes antiguas de la ciudad, con el objetivo de que los usuarios o personas que transiten fuera, observen el registro de un momento capturado de la historia de la ciudad y comparar el desarrollo de la misma en el tiempo (ver figura 1). 


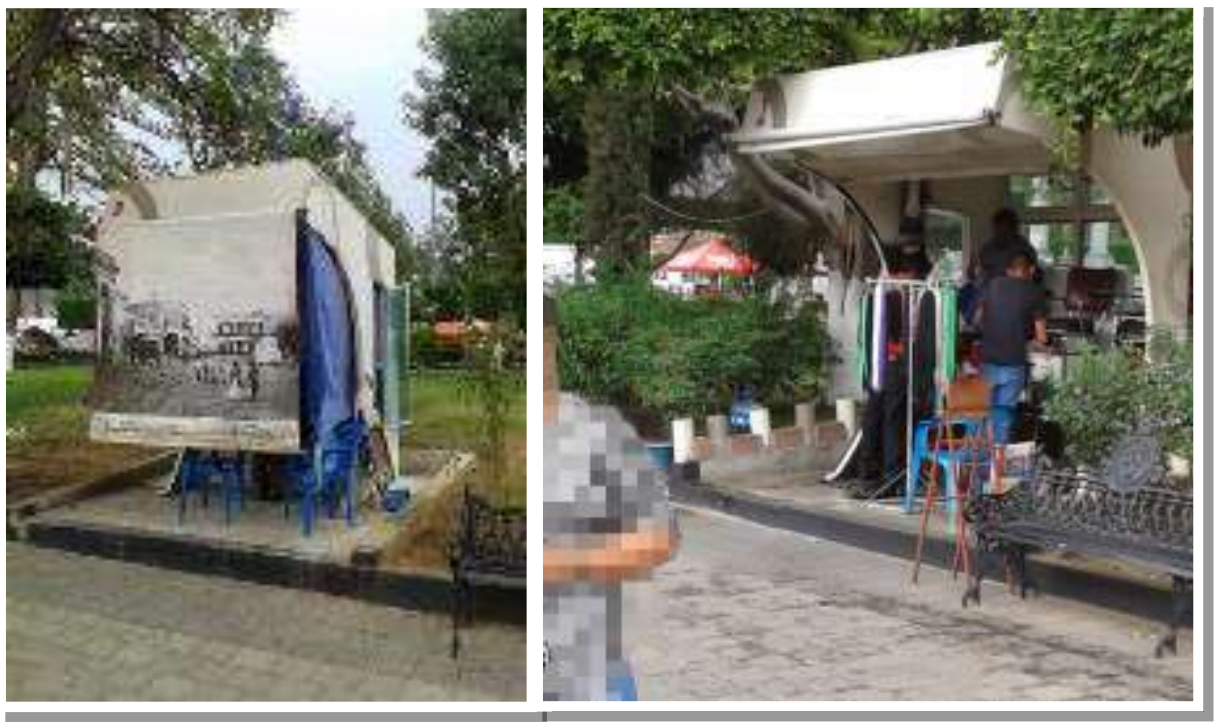

Fig. 1. Comparativa a través del tiempo de los puestos de servicios.

Un aspecto interesante observado, es el equipamiento de cada puesto de servicio para la atención al usuario, en donde el 80 por ciento de los boleros observados, disponen de revistas o periódicos, un 15 por ciento en otras casetas cuentan con radio e incluso en la parte superior de la caseta se encuentra una televisión y solo un 5 por ciento incorporan los anteriores e incluyen otros como venta de productos de calzado, golosinas e incluso bebidas embotelladas. Lo que fue observable, en aquellos boleros que incluyen otros servicios, es la búsqueda de elementos alternos de entretenimiento o distintivos de sus servicios para captar la atención y preferencia de sus clientes.

El tiempo promedio registrado de dicho servido es de entre 7 y 10 minutos aproximadamente, en fuerte dependencia al estado y dimensiones del calzado de cada usuario, así como otros servicios complementarios como lavado, pintado o reparaciones superficiales.

En un horario de 4 a 7 de la tarde, durante un día domingo, el grupo observable de clientes, se ubicaron entre los 30 y 50 años de edad, todos ellos varones, recurrieron durante dicho lapso, a los puestos de boleros para la prestación del servicio. Se encontraron 2 puestos de servicio cerrados y los que permanecieron abiertos se tuvo un registro de 10 servicios promedio. Algunos clientes mantenían en mano una revista o periódico durante el servicio, algunos escuchaban la radio, como otros observaban la televisión (en aquellos puestos que contaban con el servicio), el resto optó simplemente en conversar con los prestadores del servicio.

En lo referente a los boleros, se observó que todos ellos son hombres, jóvenes mayores de 25 años y señores de más 60 años aproximadamente. Entre las características notables de su oficio, resalta su aplicación ágil y sistémica, dado que la adquisición de sus habilidades bien puede ser resultado mismo de sus destrezas 
psicomotoras y su relación con los años de experiencia en el oficio, algunos desde muy temprana edad.

Como estos datos, se percibió que dichos puestos de servicios, pueden ser un punto importante y en beneficio del turismo local y a la competitividad comercial de los prestadores de servicios, ya que los clientes son personas en edad productiva y con potencial adquisitivo, dado entonces que el tiempo que invierten al tomar un servicio de limpieza para su calzado puede ser utilizado para brindar una prestación alternativa de entretenimiento o esparcimiento, la cual puede colaborar al incremento en sus clientes y se manifieste en un conveniente desarrollo económico local de los involucrados, a través de la difusión de los diversos destinos turísticos y culturales de la región que se traduzca en la estimulación de la actividad turística y económica del estado, con la visita de más turismo nacional e internacional, al permitir incluir en los recorridos virtuales a empresas tradicionales, tales como de comida típica como de productos artesanales.

\section{Diseño del prototipo}

Los prototipos son una manera efectiva de probar y validar la funcionalidad de una propuesta de diseño, antes de avanzar en el desarrollo. Los prototipos también son una gran manera de experimentar e idear con el dispositivo y/o los usuarios, sobre todo cuando se trata de lidiar con interacciones complejas [4].

El prototipo propuesto es un Software de Recorrido Turístico-Cultural Virtual, utilizando un dispositivo de hardware de Realidad Virtual.

Para ello se creó un prototipo de mediana fidelidad en papel, los cuales son útiles ya que se pueden crear tantos escenarios como se necesiten. También se pueden revisar rápidamente de una prueba a otra, es decir, si un usuario potencial dice un error evidente en algo que ha creado, no es un proceso complejo para actualizar el diseño antes de avanzar en el desarrollo. También es barato, más allá de la cantidad de tiempo que se invierte en la elaboración de prototipos de papel, generalmente puede se puede crear cualquier escenario por un costo menor de lo esperado [4].

Por tanto, las vistas generales del dispositivo se encuentran en la Fig. 2, mostrando el visor de Realidad Virtual de color negro, con una diadema ajustable, que se adapta al tamaño de la cabeza de cualquier usuario.

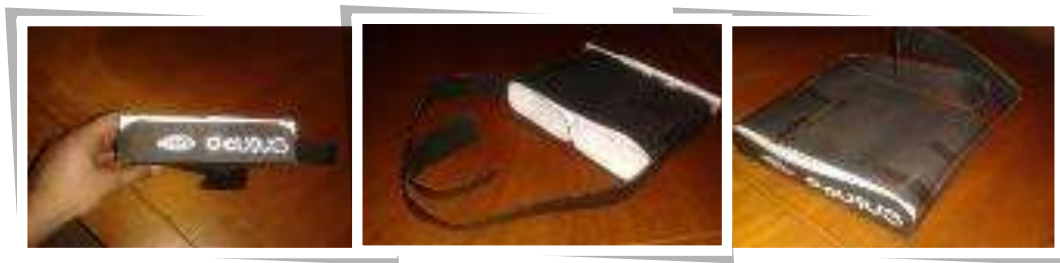

Fig. 2. Prototipo de mediana fidelidad de Realidad Virtual.

Para completar el prototipo, además de hardware se diseñó el software que acompaña al visor, el cual propone la utilización de geolocalización utilizando el API de Google Maps, el diseño de interfaz cuenta con una estructura minimalista para facilitar la 
navegación y el recorrido virtual, el usuario puede girar hacia los costados, arriba y hacia abajo con solo girar su cabeza .

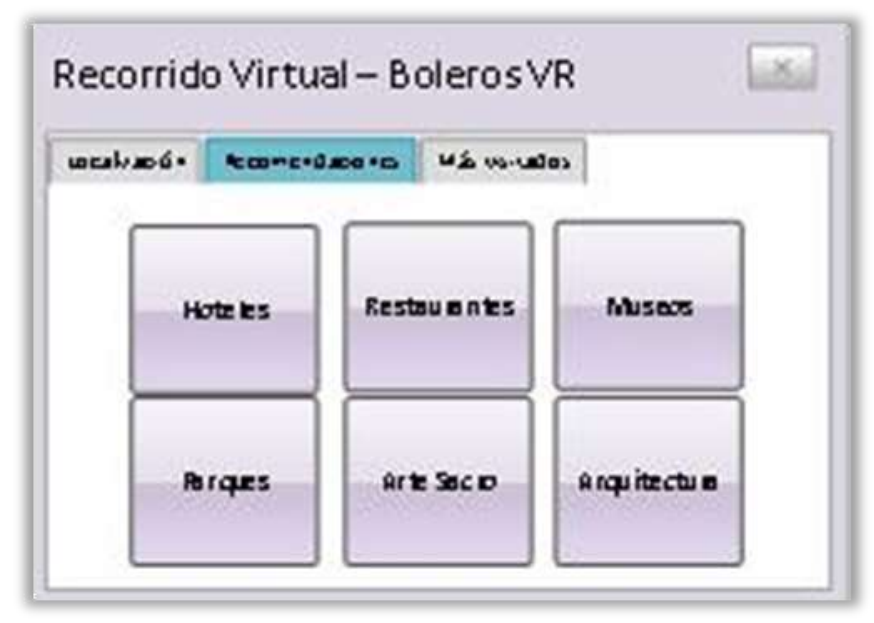

Fig. 3. Software de paseo virtual.

La aplicación muestra la pantalla inicial, con las opciones Localización, Recomendaciones y más visitados (ver Fig. 3).

El menú de recomendaciones muestra en pantalla diferentes opciones las cuales son clasificaciones de los sitios posibles de visitar filtrando por categorías, como Hoteles, Restaurantes, Museos, Parques, Arte Sacro y Arquitectura.

Finalmente, el menú de más visitados, permite la visualización de una pequeña galería con los sitios más visitados en la aplicación, esto permite al usuario, valorar su selección en base a la preferencia de otros usuarios de la aplicación.

\section{Evaluación del prototipo}

Para medir la aceptación y facilidad de uso del prototipo, se realizó una evaluación de usabilidad.

\subsection{La muestra}

La evaluación incluyó la participación de 16 estudiantes del Departamento de Música del Instituto Universitario de Bellas Artes de la Universidad de Colima, son individuos hombres y mujeres entre los 16 a los 21 años. Entre su perfil de competencias, se encuentran las tecnológicas aplicadas a la producción musical y multimedia. 


\subsection{Aplicación de la prueba}

La sesión, tuvo una duración de 1 hora con 40 minutos aproximadamente, donde cada participante realizó la prueba, con muy pocas incidencias, las cuales consistieron en detalles como el desconocimiento del concepto de Realidad Virtual, incomprensión en algunos términos del formato de consentimiento así como el replanteamiento de algunos otros conceptos de los instrumentos pre-prueba, como por ejemplo: dejar en blanco algunas otras preguntas si su respuesta no era positiva.

Durante el inicio de la sesión, previo a la implementación de la prueba, se le describió individualmente a los participantes la actividad que iba a realizar, así también se les solicitó el llenado de un cuestionario pre-prueba o de antecedentes, con el objetivo de conocer su experiencia previa con tecnologías relacionadas al prototipo sujeto de pruebas. Los temas de los reactivos buscan reconocer de forma general: su experiencia con tecnologías 3D, videojuegos con algunas tecnologías de sensores o realidad aumentada.

Una vez finalizado la fase del cuestionario previo, se llevó a cabo la evaluación al prototipo, a través de una técnica llamada "Mago de Oz", la cual ha llegado a ser de uso común en los campos de la psicología experimental, factores humanos, ergonomía y usabilidad de ingeniería para describir una prueba o metodología de diseño iterativo en el que un experimentador (el "Mago"), en un laboratorio, simula el comportamiento teórico de una aplicación informática [5].

Posteriormente, a los participantes se les solicitó contestar dos cuestionarios: la Escala de Usabilidad para Sistemas y el Modelo de Aceptación de la Tecnología.

La Escala de Usabilidad para Sistemas (SUS), ofrece una herramienta confiable para medir la usabilidad. Consiste en un cuestionario de 10 elementos con una escala Likert de cinco opciones de respuesta para los encuestados [6].

Para medir la aceptación de la plataforma se utilizó el Modelo de Aceptación Tecnológica (TAM) desarrollado por [7]. El propósito del TAM es explicar las causas de la aceptación de la tecnología por parte de los usuarios. Propone que las percepciones de un individuo en la utilidad percibida y la facilidad de uso percibida de un sistema de información, sean concluyentes para determinar su intención para usar dicho sistema.

\section{Resultados}

La evaluación generó los siguientes resultados.

\subsection{Cuestionario de antecedentes}

El propósito de este instrumento es analizar a que nivel el usuario se encuentra involucrado con el entorno donde el prototipo se implementará, y a qué grado reconoce la tecnología a que fue expuesto.

La muestra, compuesta por 13 hombres (81.25\%) entre los 16 a 21 años de edad, en contraste con las 3 mujeres (18.75\%) entre los 16 y 17 años de edad que componen una población de 16 participantes encuestados. 
Los datos de la pre-prueba arrojaron los siguientes datos:

El $62.5 \%$ de los participantes expresaron que sí han realizado algún tipo de recorrido o visita de índole turístico o cultural, el $40 \%$ de ellos lo hicieron hace menos de un año, otro $40 \%$ entre uno y cinco años y otro $20 \%$ hace más de cinco años. Por otro lado el resto de la población no ha realizado visita de estas características.

Al consultarles sobre si en alguna ocasión han utilizado algún sistema informático para realizar algún tipo de recorrido turístico y/o cultural el $81 \%$ contestó que No y el $19 \%$ contestó que Sí.

Por último, sobre su conocimiento del alguna tecnología de Realidad Virtual expresamente para recorridos turísticos y/o culturales, el 38\% respondieron que Sí, contra $62 \%$ que manifestaron que No. De tal forma, que las tecnologías que resultaron conocidas por los participantes que respondieron afirmativamente son las siguientes:

- Cabinas Simuladores de Parques Temáticos y Zoológicos.

- Tecnología de Google.

- Oculus Rift en la exploración espacial.

\subsection{Escala de Usabilidad para Sistemas (SUS)}

De acuerdo a los resultados obtenidos de la aplicación del instrumento SUS, se obtuvieron datos sobre una calificación en una escala de 0 a 100 , donde 0 hace referencia a la ausencia de usabilidad, y por el contrario, 100 como de muy alta usabilidad. La evaluación por parte de los usuarios dio una calificación de 76.3. En la Fig. 4 se puede encontrar la distribución de las frecuencias. Debido a lo anterior podemos decir que el prototipo cuenta con una usabilidad aceptable, ya que está arriba del rango indicado (70) por el autor de la escala.

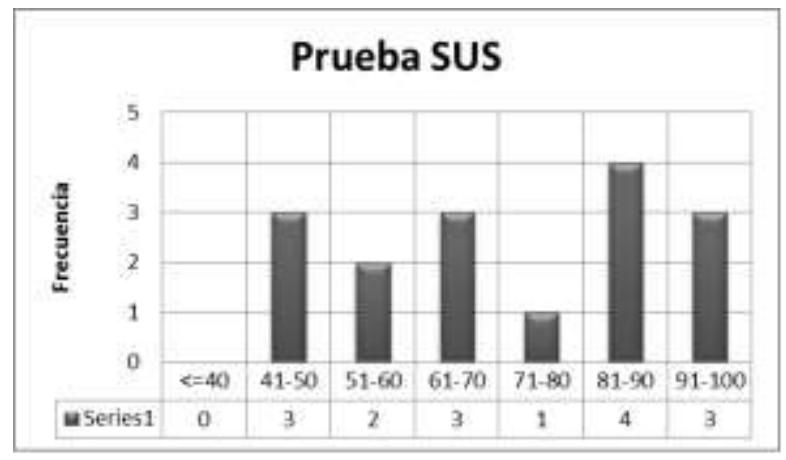

Fig. 4. Resultados SUS.

\subsection{Modelo de Aceptación de la Tecnología (TAM)}

Como se mencionó previamente, su principal objetivo es describir las razones de la aceptación o rechazo por parte del usuario sobre un objeto de tecnología, por lo que sugiere que la percepción de utilidad y facilidad de uso en un sistema son suficientes para determinar la intensión de utilización de la tecnología evaluada. 
En la percepción de facilidad de uso, el $87.5 \%$ consideró que es fácil de usar, el $81.3 \%$, opinó que es sencillo de aprender, el $87.5 \%$ pensó que es claro y otro porcentaje igual al anterior opinó que era fácil de navegar. Como se muestra en la Fig. 5 , el porcentaje de rechazo en este concepto en general solo representó el $14.1 \%$ contra un $85.9 \%$ que opinó positivamente.

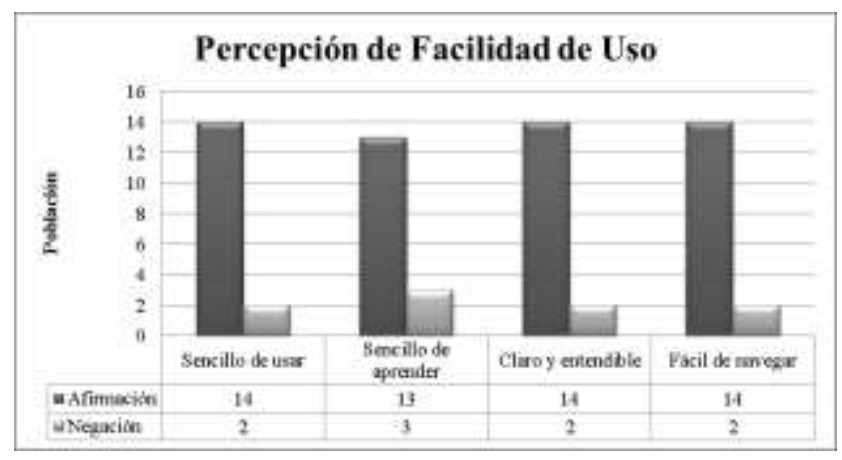

Fig. 5. Facilidad de Uso.

De acuerdo a los resultados (ver Fig. 6), sobre el concepto de Percepción de Utilidad a la precepción hacia el uso del software donde un 100\% percibió Eficiencia del software, además que otro porcentaje igual consideraron Satisfacción, el 87.5\% estuvo de acuerdo en la productividad del software y el total de los encuestados afirmó estar de acuerdo con su utilidad.

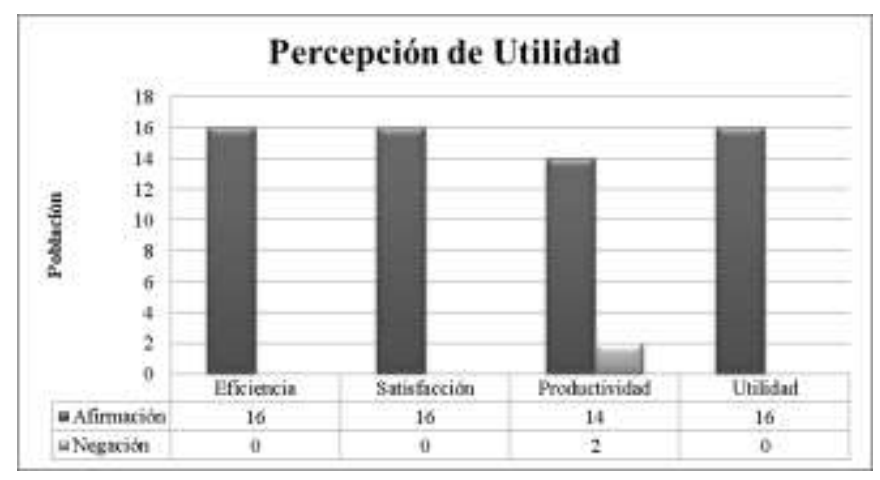

Fig. 6. Utilidad.

Los resultados, sobre su Actitud hacia el uso del software (ver Fig. 7), un 100\% percibió a disgusto del software y otras cantidades iguales con los siguientes conceptos, lo que demuestra muy probablemente incomprensión en este aspecto, por lo que los datos no son significativos. 


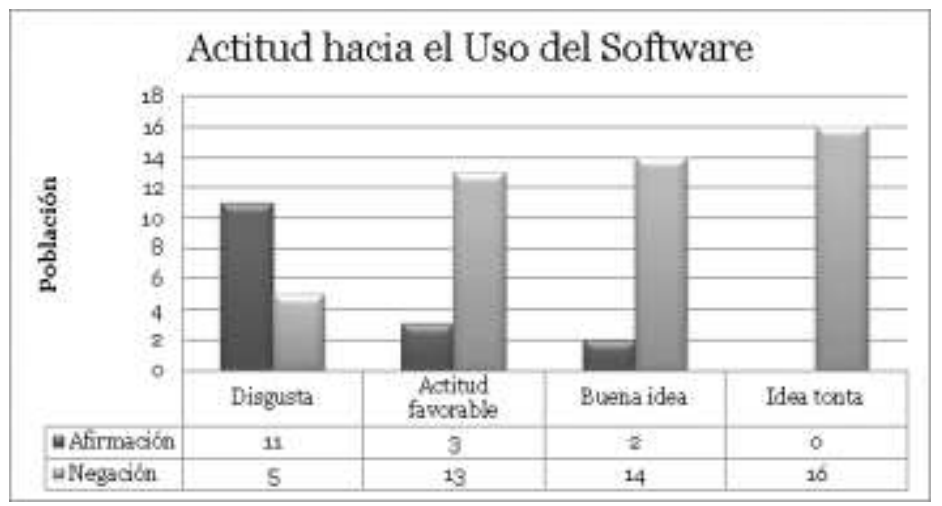

Fig. 7. Actitud de Uso.

Finalmente, sobre la Intensión de Uso, los encuestados, contestaron de forma unánime, con un $100 \%$, su total acuerdo sobre su intención de volverlo a usar y la decisión de usar este software en sus visitas o recorridos turísticos-culturales (ver Fig. 8).

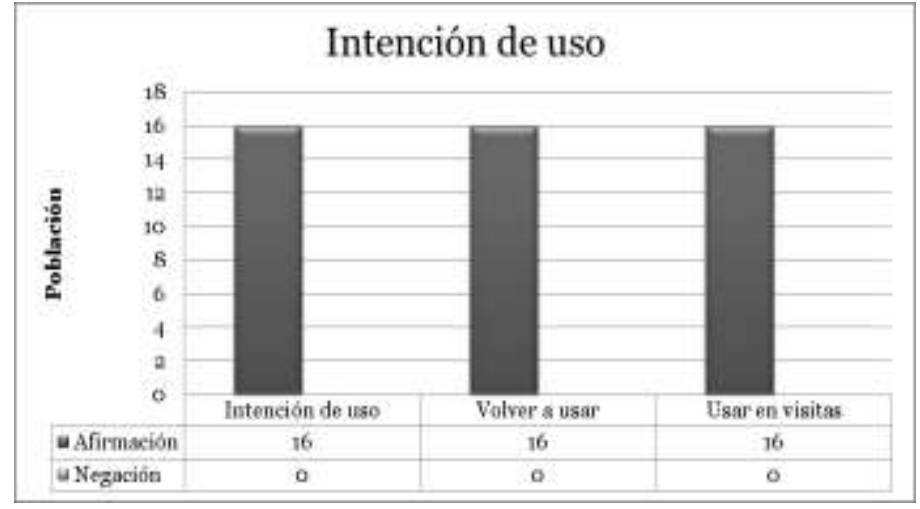

Fig. 8. Intención de Uso.

\subsection{Propuestas de mejora}

Algunas de las observaciones de los participantes se manifestaron en ideas consistentes en incorporar además de recorridos de índole turístico, la posibilidad de incorporar simuladores, como de aeronaves u otros vehículos con movimiento, esto con la finalidad de darle un valor agregado en el aspecto de entretenimiento. 


\section{Conclusiones}

Este trabajo presenta un prototipo basado en Realidad Virtual para recorridos turístico-culturales, la evaluación de este prototipo dio resultados muy similares entre los instrumentos de evaluación aplicados, por un lado la prueba SUS nos permitió determinar con bastante certeza que nuestro prototipo cuenta con un grado de usabilidad aceptable.

La prueba TAM, permitió definir muy claramente la posición de los usuarios, con muy buen grado de aceptación de la tecnología propuesta. Así mismo esta prueba permitió recibir retroalimentación sobre las áreas de mejora, como la utilización de algún tipo de audio para acercarse lo más posible a la realidad de la propuesta, así como detalles ergonómicos del prototipo en papel, como el largo de la correa y falta de una mejor iluminación, dada la sombra que da la propia forma interna del prototipo. Sin embargo el diseño de pase de diapositivas sobre las gafas significó una experiencia novedosa para los participantes, lo que provocó mayor retroalimentación de su parte hacia la prueba.

En cuanto a fortalezas, finalmente, el soporte de hardware del prototipo, presentó una completa aceptación en la explicación de su funcionamiento y de las posibilidades de este, por ello estas tecnologías mostraron ser muy atractivas a los usuarios encuestados. Por tanto, el prototipo presenta un buen grado de aceptación en un entorno real, lo que claramente justificaría continuar con el desarrollo de las siguientes fases del proyecto.

\section{Agradecimientos}

Agradecemos a Julia Elena Nolazco Nuñez y a Héctor Torres López, alumnos de la Maestría en Tecnologías de Información de la Facultad de Telemática, por el apoyo otorgado en el desarrollo del prototipo usado en el presente trabajo, desarrollo creado durante la materia de Interacción Humano-Computadora.

\section{Referencias}

1. Martínez, L. (2009). Presentación. Tecnologías de la información I: guía de aprendizaje para el bachillerato general por competencias (p. 10). Guadalajara, Jal.: Universidad de Guadalajara, Editorial Universitaria.

2. Instituto Andaluz del Patrimonio Histórico. (2003). El patrimonio virtual: alcance y desafío. Revista PH.

3. González, E., \& Martínez, M. I. (2002, Feb. - Mar.). La contribución al empleo de las Nuevas Tecnologías de la Información y Comunicación (NTIC). Revista Economistas, No. 91, 267-276.

4. Unger, R., \& Chandler, C. (2012). 13. Prototyping: Breathing (Some Sort of) Life into Your Designs. A project guide to UX design: for user experience designers in the field or in the making (2nd ed., p. s/n). Berkeley, Calif.: New Riders). 


\section{Gabriel Peralta y Pedro Santana-Mancilla}

5. Kelley, J. (n.d.). Wizard of $\mathrm{Oz} \mid$ Usability Body of Knowledge. Wizard of Oz | Usability Body of Knowledge. Retrieved June 12, 2014, from http://www.usabilitybok.org/wizardof-oz.

6. Brooke, J. (1996). SUS: a 'quick and dirty' usability scale. En Usability evaluation in industry (pág. 189). UK: Taylor\&Francis.

7. Davis, F. D. (1989), "Perceived usefulness, perceived ease of use, and user acceptance of information technology", MIS Quarterly, 13(3): 319-340. 